\title{
Versatile supercontinuum generation by using $x(2)$ and $x(3)$ nonlinearities in PPLN crystal for direct multiplex CARS measurement
}

Wehbi, Sahar, Mansuryan, Tigran, Jauberteau, Raphael, Tonello, Alessandro, Krupa, Katarzyna, et al.

Sahar Wehbi, Tigran Mansuryan, Raphael Jauberteau, Alessandro Tonello, Katarzyna Krupa, Stefan Wabnitz, Hideaki Kano, Philippe Leproux, Sebastien Vergnole, Vincent Couderc, "Versatile supercontinuum generation by using $X$ (2) and $X(3)$ nonlinearities in PPLN crystal for direct multiplex CARS measurement," Proc. SPIE 11770, Nonlinear Optics and Applications XII, 1177017 (18 April 2021); doi: 10.1117/12.2589155

SPE. Event: SPIE Optics + Optoelectronics, 2021, Online Only 


\title{
Versatile supercontinuum generation by using $\chi^{(2)}$ and $\chi^{(3)}$ nonlinearities in PPLN crystal for direct multiplex CARS measurement
}

\author{
Sahar Wehbi ${ }^{1,2}$, Tigran Mansuryan ${ }^{1}$, Raphael Jauberteau ${ }^{1}$, Alessandro Tonello ${ }^{1}$, Katarzyna \\ Krupa $^{3}$, Stefan Wabnitz ${ }^{4,5}$, Hideaki Kano ${ }^{6,7,8}$, Philippe Leproux ${ }^{1}$, Sebastien Vergnole ${ }^{2}$ and \\ Vincent Couderc ${ }^{1}$ \\ ${ }^{1}$ University of Limoges, XLIM, UMR CNRS 7252, 123 Avenue A. Thomas, 87060 Limoges, \\ France \\ ${ }^{2}$ ALPhANOV, Optics \& Lasers Technology Center, Institut d'Optique d'Aquitaine, Rue François \\ Mitterrand, 33400 Talence, France \\ ${ }^{3}$ Institute of Physical Chemistry, Polish Academy of Sciences, 01-224 Warsaw, Poland \\ ${ }^{4}$ DIET, Sapienza University of Rome Via Eudossiana 18, 00184 Rome, Italy \\ ${ }^{5}$ CNR-INO, Istituto Nazionale di Ottica, Via Campi Flegrei 34, I-80078 Pozzuoli (NA), Italy \\ ${ }^{6}$ Department of Applied Physics, Graduate School of Pure and Applied Sciences, University of \\ Tsukuba, 1-1-1 Tennodai, Tsukuba, Ibaraki 305-8573, Japan \\ ${ }^{7}$ Institute of Applied Physics, University of Tsukuba, 1-1-1 Tennodai, Tsukuba, Ibaraki 305-8573, \\ Japan \\ ${ }^{8}$ Department of Chemistry, Kyushu University, 744, Motooka, Nishi-ku, Fukuoka-shi, Fukuoka \\ 819-0395, Japan
}

\begin{abstract}
In this study, we report on experiments of spatio-temporal nonlinear frequency conversion in a periodically poled Lithium Niobate (PPLN) crystal designed for second-harmonic generation (SHG). We demonstrated a novel supercontinuum source based on the mixing of second and third-order nonlinearities. We could adjust the $\left(\chi^{(2)}, \chi^{(3)}\right)$ nonlinearities by controlling the input laser polarization orientation, the pulse duration and the PPLN crystal temperature. We obtained an ultra-broadband spectrum, ranging from visible to infrared domains, by pumping a 20-mm-long PPLN crystal with a 3 ps pulse at $1030 \mathrm{~nm}$. This broadband pulse was used to achieve direct multiplex Coherent Anti-Stokes Raman Scattering (M-CARS) imaging, without the need for any optical delay line to temporally synchronize the pump and the Stokes waves. Simultaneous vibrational signatures ranging from $-3200 \mathrm{~cm}^{-1}$ to $-500 \mathrm{~cm}^{-1}$ were obtained. Several filters were placed on the broadband supercontinuum path, to shape the output spectrum between $1030 \mathrm{~nm}$ and $1650 \mathrm{~nm}$, before sending it into the microscope. The output spectral analysis allows for the demonstration of multimodal imaging, by using SHG, M-CARS and multiphoton fluorescence processes.
\end{abstract}

Keywords: Nonlinear optics, Nonlinear crystal, Supercontinuum, Multiplex CARS.

\section{INTRODUCTION}

The CARS technique is a nonlinear process, which combines the effects of Raman scattering and four-wave-mixing (FWM). A pump, a probe and a Stokes (monochromatic) waves interact with a sample, generating an anti-Stokes wave that contains information about molecular vibrations. To excite several vibrational modes, a monochromatic "Stokes" wave can be replaced by a polychromatic beam [1-2]. This so-called multiplex-CARS (M-CARS) method requires the development of supercontinuum (SC) laser sources. Indeed, many SC sources have been obtained by using photonic crystal fibers (PCFs) exploiting their third-order nonlinearities. In that case, the input laser was split into two parts: a pump beam and a Stokes beam (which was generated by exploiting Raman scattering in a PCF). These beams were temporally re-synchronized on the sample by the help of an optical delay line placed on the pump beam path. One of the 
challenges in M-CARS is to simplify the experimental setup by removing the optical delay line: this can be achieved by replacing PCFs with nonlinear crystals.

SC generation, obtained either with crystals or with optical fibers, is a complex process that involves several nonlinear effects (Self-phase modulation, four wave-mixing, soliton propagation, cascading effect, ...). SC is typically obtained by pumping a nonlinear medium with short optical pulses (with durations ranging from several hundred of femtoseconds to several hundred of picoseconds). In this study, we will investigate the generation of an ultra-broadband spectrum in PPLN crystal, pumped with a picosecond laser. We will demonstrate that although the PPLN crystal which is commonly used for SHG, it can also provide efficient SC generation. Moreover, we show that the shape of the SC could be modified in real time, by adjusting the second and the third order nonlinearity amounts. The resulting SC is exploited as the Stokes beam in our M-CARS measurements. It should be underlined that the SC Stokes beam was directly synchronized with the pump beam, thanks to the short length of the PPLN crystal (20 mm).

\section{EXPERIMENTAL SETUP}

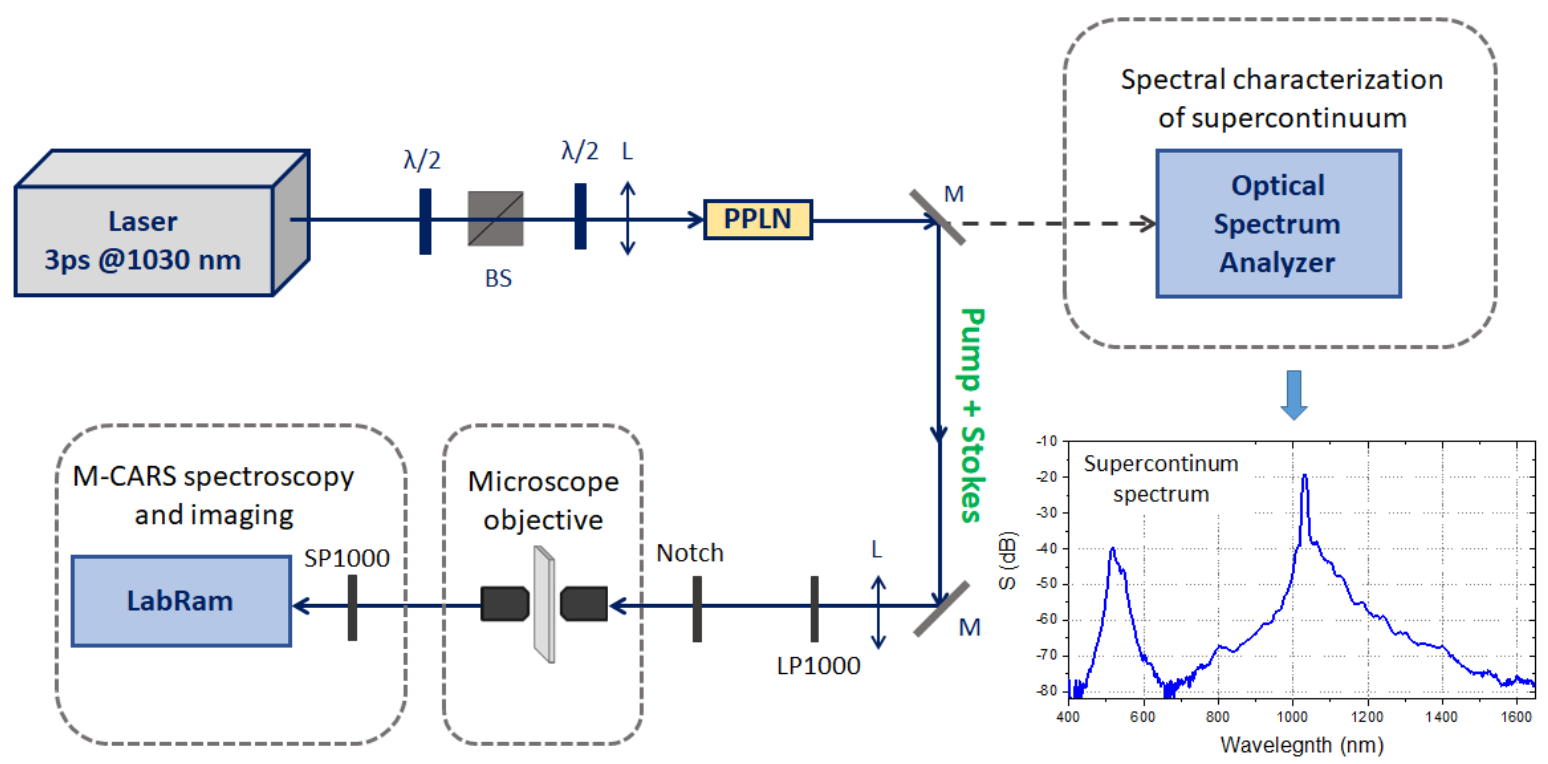

Figure 1: Experimental setup used to generate SC in a PPLN crystal and the M-CARS measurement. $\lambda / 2$ : half-wave plate; BS: polarizing beam splitter; L: lens; M: mirror; LP1000: long pass filter (>1000 nm); SP1000: short pass filter $(<1000 \mathrm{~nm})$; Inset: example of a supercontinuum obtained with our setup.

Figure 1 shows our experimental setup. To pump the PPLN crystal, we used a $30 \mathrm{kHz}$ laser at $1030 \mathrm{~nm}$, delivering pulses of tunable duration. Two half-wave plates and a polarizing beam splitter were added before the crystal, to control the optical energy and the beam polarization. The output beam was split into two pathways. The first one was used to analyze the SC spectrum with an optical spectrum analyzer. The second beam was delivered via different filters to a microspectrometer, to obtain the M-CARS spectrum and the images of the samples.

\section{RESULTS}

\subsection{Supercontinuum generation}

The PPLN crystal is known for its high second harmonic generation efficiency. However, recent studies have shown that, a broadband spectrum can be generated especially in the phase-mismatched conditions, with a temperature higher than the phase-matching temperature. This broadband spectrum is due to the modulation instability and the cascading effects 
in a PPLN crystal exploiting the second order nonlinearity $\chi^{(2)}$ [3] (see figure 2). A rotation of the linear input polarization can drastically reduce the quadratic process which reshapes the supercontinuum in the visible and infrared domains. Because of its high third-order nonlinear susceptibility, the PPLN crystal can modify the initial spectrum of the pump beam and produce symmetric broadening between $800 \mathrm{~nm}$ and $1600 \mathrm{~nm}$ (see figure 1). Thus, the nonlinear process at the origin of the supercontinuum is mainly due to the self-phase modulation for an input polarization direction oriented close to $90^{\circ}$ with respect to the first experiment. An intermediate position of the polarization direction (between 0 and $90^{\circ}$ ) can introduce an interplay between second and third-order nonlinearities and provides an easy control of the spectrum profile (see figure 2). The visible part of the supercontinuum ranging from $450 \mathrm{~nm}$ to $620 \mathrm{~nm}$ is due to quadratic conversion of the infrared spectrum by means of a remaining second harmonic generation within a type I process obtained with a large phase mismatch configuration.

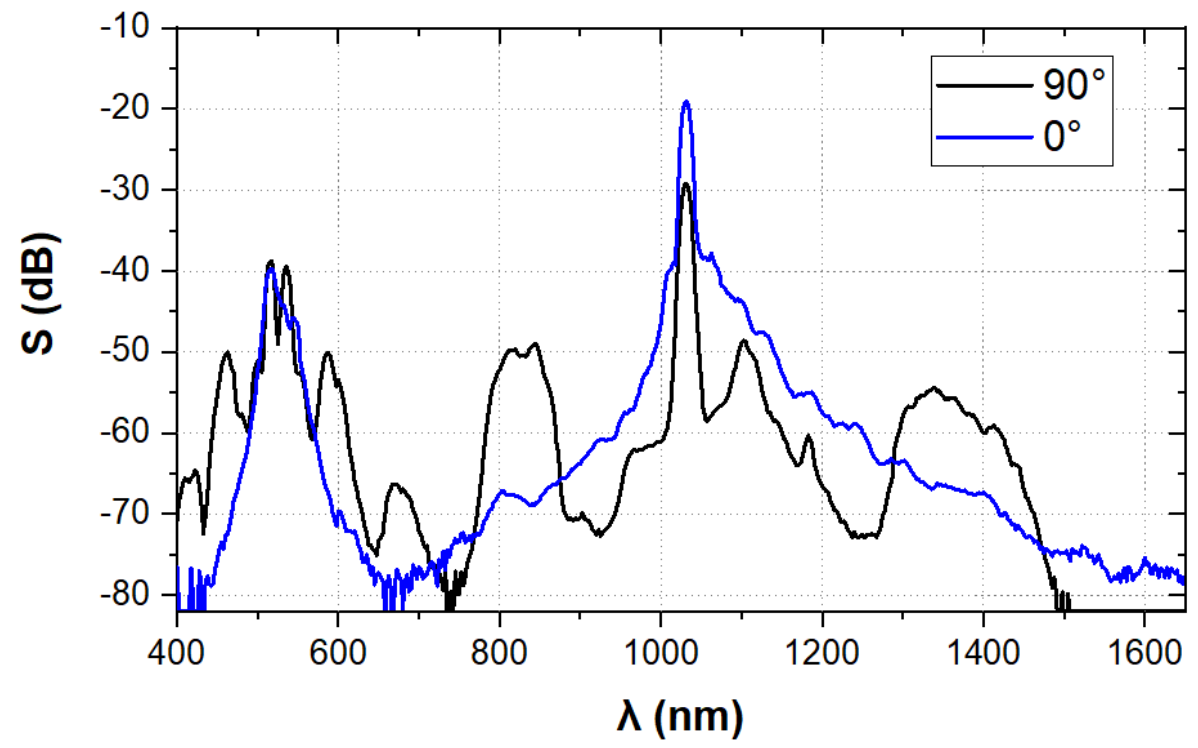

Figure 2: SC spectra generated in a PPLN crystal for two orientations of the input polarization $\left(0^{\circ}-90^{\circ}\right)$, pulse duration: 3 ps.

\subsection{Impact of the pulse duration and the crystal temperature}

We investigated the impact of the pulse duration and the temperature on the generation of the supercontinuum, which can be used as the pump and the Stokes waves in M-CARS measurements. We pumped the PPLN crystal with optical pulses ranging from $270 \mathrm{fs}$ and up to $100 \mathrm{ps}$ with a constant pulse energy. The temperature of the PPLN crystal was fixed at $200^{\circ} \mathrm{C}$ which corresponds to a second harmonic process obtained with a large phase mismatch conditions (Phase matching temperature: $41^{\circ} \mathrm{C}$ ). Thus, the spectrum ranges from $450 \mathrm{~nm}$ to $1600 \mathrm{~nm}$ with a minimum at $700 \mathrm{~nm}$. The upper part $(800 \mathrm{~nm}-1600 \mathrm{~nm})$ of the SC exhibits a triangular shape for short pulse duration i. e. between $250 \mathrm{fs}$ and $3 \mathrm{ps}$. Indeed, in that configuration, the group velocity mismatch becomes larger and no longer superimposition between the fundamental and the second harmonic is obtained. Then, the SC is mainly due to the Kerr effect by means of self-phase modulation. By increasing the pulse duration, the shape of the SC is changed and nonlinear conversions between 1200 $\mathrm{nm}$ and $1400 \mathrm{~nm}$ are clearly visible. Therefore, for very long pulse (100 ps), the peak power drastically falls, and no spectral broadening is observed (fig. 3(a)). 

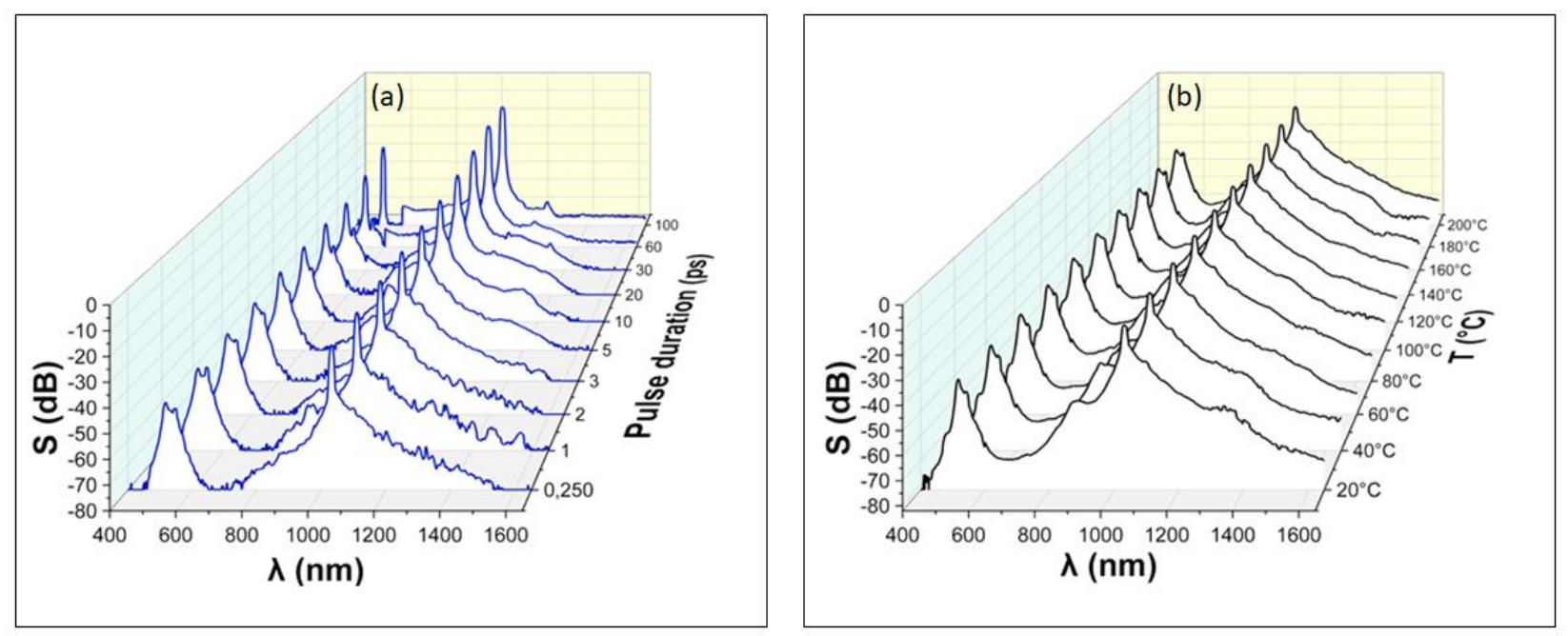

Figure 3: (a) Evolution of SC generated in a PPLN crystal vs. input pulse duration for a temperature of $200^{\circ} \mathrm{C}$ and a laser repetition rate of $30 \mathrm{kHz}$, (b) vs. temperature for an optical pulse duration of $3 \mathrm{ps}$.

Next, we studied the effects of the variation of the PPLN temperature on SC generation. For an optical pulse duration of 3 ps, we varied the PPLN temperature from $20^{\circ} \mathrm{C}$ to $200^{\circ} \mathrm{C}$. The evolution of the SC spectrum is shown in Figure $3(\mathrm{~b})$. As observed the spectral shape of the SC does not evolve so much versus the temperature which demonstrates that the main spectral broadening is driven by the Kerr effect. However, lateral shoulders are visible for temperature close to the phase matching configuration $\left(41^{\circ}\right)$, but progressively disappear for higher phase mismatch configurations. Thus, the SC maintains its spectral extension ranging from $450 \mathrm{~nm}$ and up to $1650 \mathrm{~nm}$.

\subsection{Multiplex CARS measurement}

In our configuration, the pump and the SC Stokes waves are directly obtained from the initial beam coupled into the PPLN crystal. Thus, and because of the low impact of the dispersion along the propagation in the nonlinear crystal, all the waves remain temporally synchronized. The SC spectrum was re-shaped by using different filters, to remove the visible part of the SC (useless and disturbing for the M-CARS signals), and to narrow the pump bandwidth (thus enhancing the spectral resolution of CARS). The polychromatic beam is sent into the microscope and is focused onto the sample with a 60X objective. The resulting forward M-CARS signals are collected through a 60X objective and then sent into a spectrometer. The measurement of a polystyrene bead demonstrates the capabilities of our approach to obtain a MCARS process. A vibrational signature of a polystyrene sample was obtained in the C-H zone at $-3055 \mathrm{~cm}^{-1}$ and simultaneously in the fingerprint region (Figure 4a). Figure $4 \mathrm{~b}$ shows the image of the polystyrene bead around -3055 $\mathrm{cm}^{-1}$ peak, with its corresponding bright-field image in Figure 4c. 

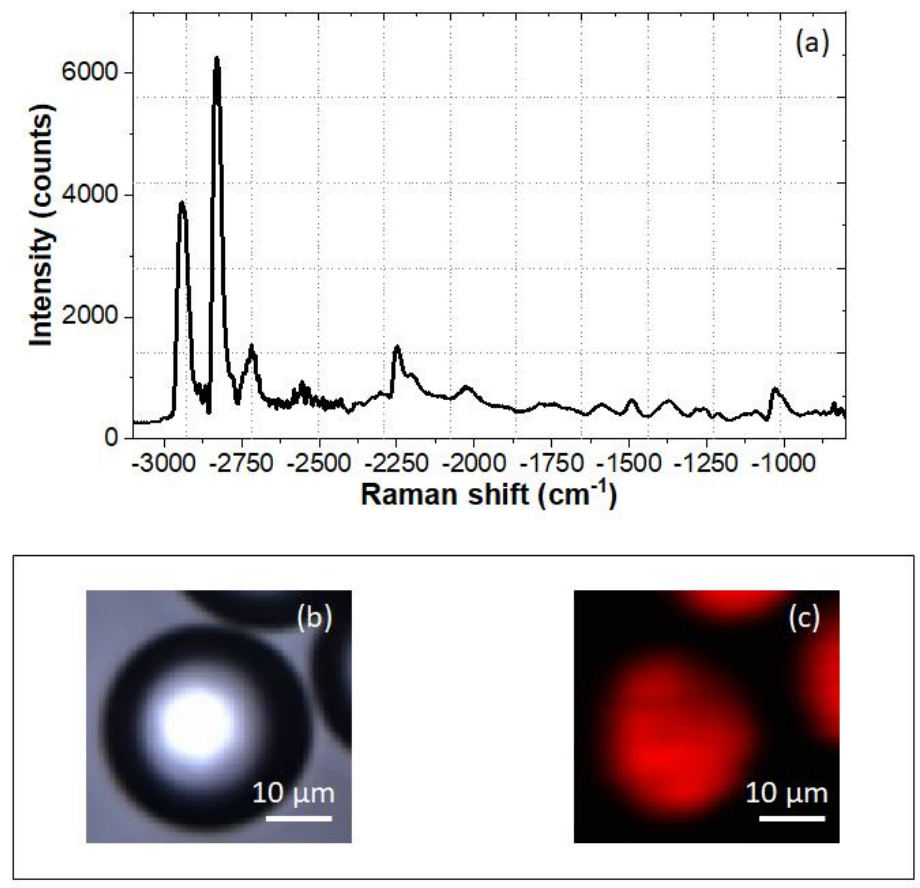

Figure 4: (a) M-CARS spectrum of methanol, (b) M-CARS image of a polystyrene bead at $-3055 \mathrm{~cm}^{-1}$ extracted from MCARS data, (c) corresponding bright-field image of the polystyrene bead.

A second series of experiments were carried out on cellulose by using two modes of imaging i.e. M-CARS process and second harmonic generation, introduced by the remaining part of the pump wave. These results are displayed on the Figure 5.
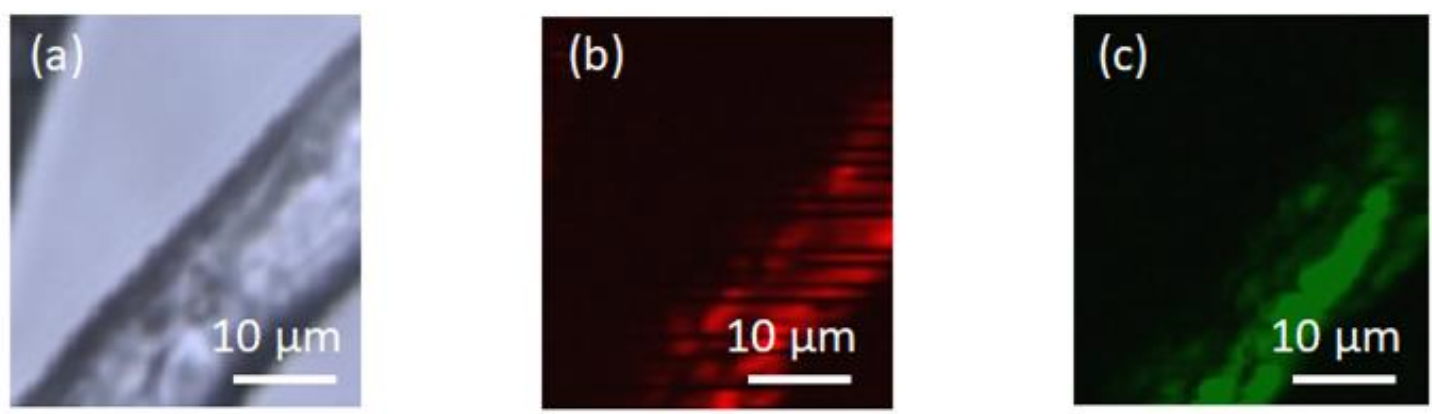

Figure 5. (a) Bright field image of cellulose fibers; (b) M- CARS image at $-2896 \mathrm{~cm}^{-1}$;(c) Second harmonic image.

\section{CONCLUSION}

We reported on SC generation in a PPLN crystal by mixing second and third-order nonlinearities. We demonstrated the possibility to control the shape of the SC spectrum by varying the input pulse duration and the optical beam polarization. The short length of the PPLN crystal allows for the generation of an ultra large spectrum, comprising directly synchronized pump and Stokes waves (used for M-CARS experiment), with no spatial degradation of the beams. Thus, 
we developed a M-CARS setup without any optical delay line. We, then, simultaneously measured the spectrum of a polystyrene bead in the $\mathrm{C}-\mathrm{H}$ and in the fingerprint regions and achieved a $2 \mathrm{D}$ image of the polystyrene bead at $-3055 \mathrm{~cm}^{-}$ ${ }^{1}$. Multimode imaging process has been demonstrated by adding an additional image at the second harmonic obtained on cellulose fibers.

\section{ACKNOWLEDGEMENT}

K. K. acknowledges the Foundation of Polish Science (TEAM-NET project No. POIR.04.04.00-00-16ED/18-00). S.W. was supported by the H2020 European Research Council (740355, 874596); V.C. acknowledge the financial support provided by: the French ANR through the "TRAFIC, project: ANR-18-CE080016-01"; the CILAS Company (ArianeGroup) through the shared X-LAS laboratory; the "Region Nouvelle Aquitaine" through the projects F2MH, the National Research Agency under the Investments for the future program with the reference ANR-10-LABX-0074-01 Sigma-LIM.

\section{REFERENCES}

[1] Champert, P.A., Couderc, V., et Barthelemy, A., «1.5-2.0- $\mu$ m Multiwatt Continuum Generation in DispersionShifted Fiber by Use of High-Power Continuous-Wave Fiber Source », IEEE Photon. Technol. Lett., 16(11), 2445-2447, (2004).

[2] Bito, K., Okuno, M., Kano, H., Tokuhara, S., Naito, S., Masukawa, Y., Leproux, P., Couderc, V., Hamaguchi, H., «Protein Secondary Structure Imaging with Ultrabroadband Multiplex Coherent Anti-Stokes Raman Scattering (CARS) Microspectroscopy », J. Phys. Chem. B, 116(4), 1452-1457, (2012).

[3] Krupa, K., Labruyère, A., Tonello, A., Shalaby, B. M., Couderc, V., Baronio, F., Aceves, A. B, « Polychromatic filament in quadratic media: spatial and spectral shaping of light in crystals », Optica, 2(12), 1058, (2015). 